\title{
EchoGéo
}

$23 \mid 2013$

Entre l'ancien et le nouveau monde. La transcendance du lieu et de l'espace en géographie vinicole contemporaine

\section{La Méditerranée au pied des Andes : l'invention des paysages viticoles dans le Cône Sud?}

\section{Cécile Faliès et Julie Le Gall}

\section{OpenEdition \\ Journals}

\section{Édition électronique}

URL : https://journals.openedition.org/echogeo/13348

DOI : $10.4000 /$ echogeo. 13348

ISSN : 1963-1197

Éditeur

Pôle de recherche pour l'organisation et la diffusion de l'information géographique (CNRS UMR 8586)

\section{Référence électronique}

Cécile Faliès et Julie Le Gall, « La Méditerranée au pied des Andes : I'invention des paysages viticoles dans le Cône Sud ? », EchoGéo [En ligne], 23 | 2013, mis en ligne le 05 juin 2013, consulté le 11 août 2021. URL : http://journals.openedition.org/echogeo/13348 ; DOI : https://doi.org/10.4000/echogeo. 13348

Ce document a été généré automatiquement le 11 août 2021.

EchoGéo est mis à disposition selon les termes de la licence Creative Commons Attribution - Pas d'Utilisation Commerciale - Pas de Modification 4.0 International (CC BY-NC-ND) 


\title{
La Méditerranée au pied des Andes : l'invention des paysages viticoles dans le Cône Sud?
}

\author{
Cécile Faliès et Julie Le Gall
}

1 Au sein de la nouvelle planète vitivinicole, l'Argentine et le Chili s'affichent comme leaders du sous-continent latino-américain en termes de superficie viticole ( $8^{\mathrm{e}}$ et $10^{\mathrm{e}}$ rangs mondiaux), de volume de vins produits ( $5^{\mathrm{e}}$ et $8^{\mathrm{e}}$ rangs mondiaux) et de volumes exportés ( $9^{\mathrm{e}}$ et $5^{\mathrm{e}}$ rangs mondiaux) (OIV, 2012). Si la culture de la vigne date dans les deux pays de la colonisation et est donc ancienne, elle a subi depuis une vingtaine d'années une transformation profonde des modes de culture et des pratiques commerciales qui justifie la position de ces États sur le marché mondial.

Comme tous les vins du Nouveau Monde, les vins du Cône Sud sont majoritairement des vins de cépage et des vins de marque, dénominations employées afin de les distinguer des vins de terroirs européens (Schirmer R., 2007). Ces caractères servent à différencier, opposer, même, des réalités culturelles et paysagères: alors que les paysages de la viticulture dans les pays européens seraient hérités d'une action humaine centenaire voire millénaire, ceux du Cône Sud seraient importés suite à l'intégration de ces vignobles dans la mondialisation économique et à l'arrivée d'acteurs et de capitaux extérieurs notamment bordelais et californiens (Roudié P., 2004). Derrière cette distinction, on présuppose que les vins de cépages et de marques sont indépendants des caractéristiques de l'espace où s'implante le vignoble et ne seraient donc pas producteurs de paysages à forte identité.

Ce numéro d'Echogéo est l'occasion de revenir sur cette idée et d'analyser les transformations et créations paysagères des vignobles du Cône Sud sous l'influence de leur intégration à la nouvelle planète des vins. Les vignobles identifiés sont ceux de Mendoza en Argentine, le principal vignoble argentin ( $32^{\circ}$ de latitude sud) et de la vallée de l'Elqui $\left(29^{\circ} \mathrm{S}\right)$, de la vallée de Colchagua $\left(33^{\circ} \mathrm{S}\right)$ et de la vallée du Maule $\left(35^{\circ} \mathrm{S}\right)$ au Chili, trois vignobles moins productifs que celui de Casablanca au centre du pays mais comptant de plus en plus dans le nouveau monde du vin. 
Illustration 1- La finca Vista Gladys, une exploitation face au piémont près de Tunuyán, Province de Mendoza, Argentine

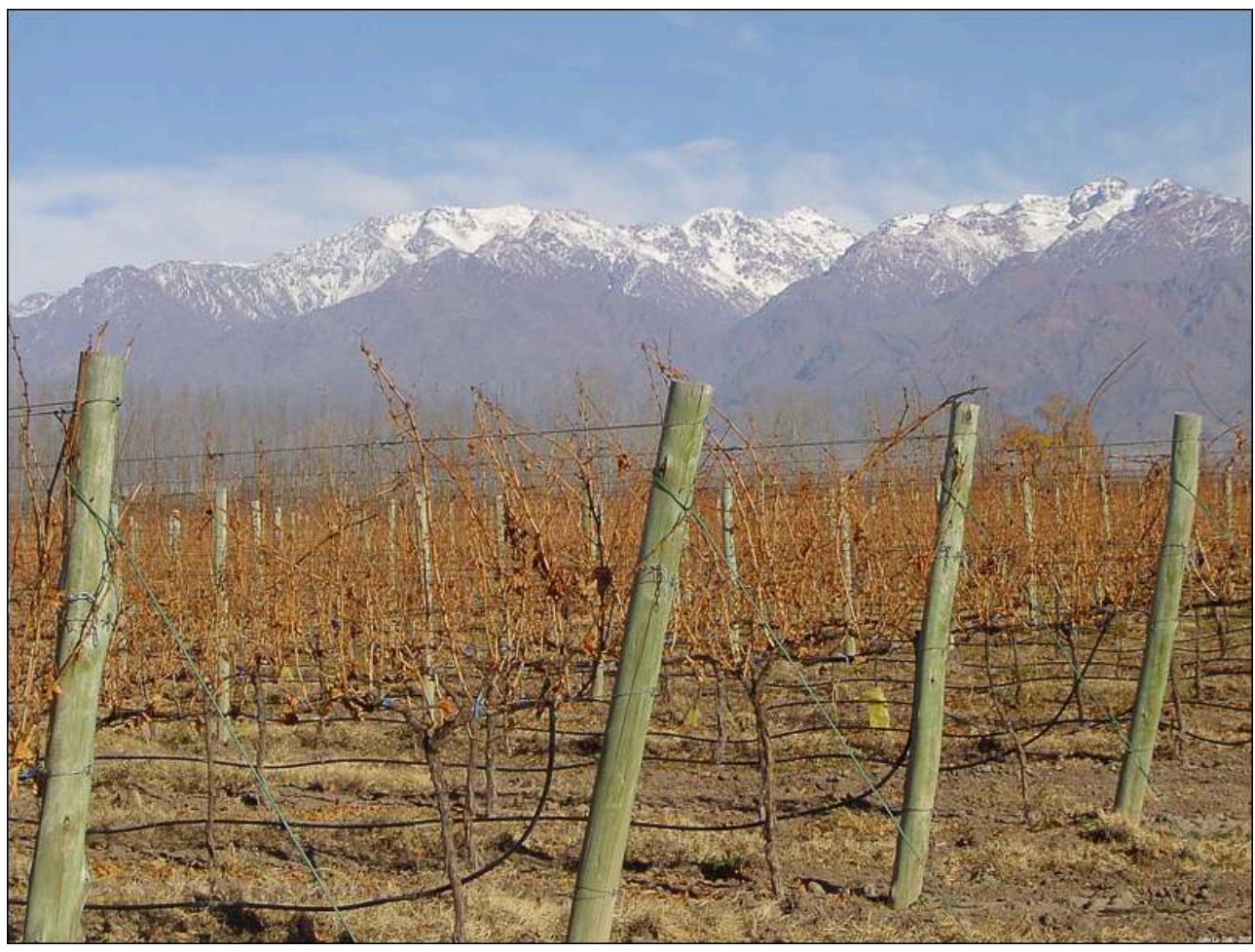

Auteur : J. Le Gall, 2006.

Difficile d'ignorer la Cordillère des Andes lors d'une visite des vignobles chiliens et argentins. L'œil ne peut se détacher du contraste entre les mers de vigne alignées dans les plaines et la massivité du piémont andin, dominé de chaque côté par l'Aconcagua, le plus haut sommet des Andes. Les saisons ne font que renforcer ces contrastes: à l'automne, camaïeux orange et rouge des vignes contre blancheur des sommets enneigés, comme sur cette photo; au printemps, vert éclatant des feuillages contre tons ocres des versants arides.

Ce cliché correspond à l'image la plus souvent véhiculée par les vignobles du Cône Sud : un vignoble de piémont aux conditions d'ensoleillement exceptionnelles, ce dont témoignent la couleur du ciel mais surtout la conduite haute et palissée de la vigne (espalderos) et la nécessaire irrigation par goutte-à-goutte des ceps. La Cordillère justifie par biens des aspects les modes de viticulture dans ces régions du monde. A l'Est, les Andes font barrière aux précipitations. Le climat désertique n'autorise la viticulture qu'au sein des trois oasis de la province de Mendoza et dans les espaces conquis grâce à une irrigation très sophistiquée.

6 Cette exploitation est celle d'une famille de la ville de Mendoza sans antécédent particulier dans les métiers du vin. Elle a investi dans cette exploitation par intérêts personnel (posséder une maison de campagne) et économique (obtenir des revenus complémentaires à partir des terres achetées). A la saison des vendanges, les raisins sont vendus à des bodegas industrielles qui participent donc à la conduite de la production afin de rendre la viticulture la plus rentable possible. 
Illustration 2 - La bodega Cheval de los Andes, propriété de Moët et Chandon, Province de Mendoza, Argentine

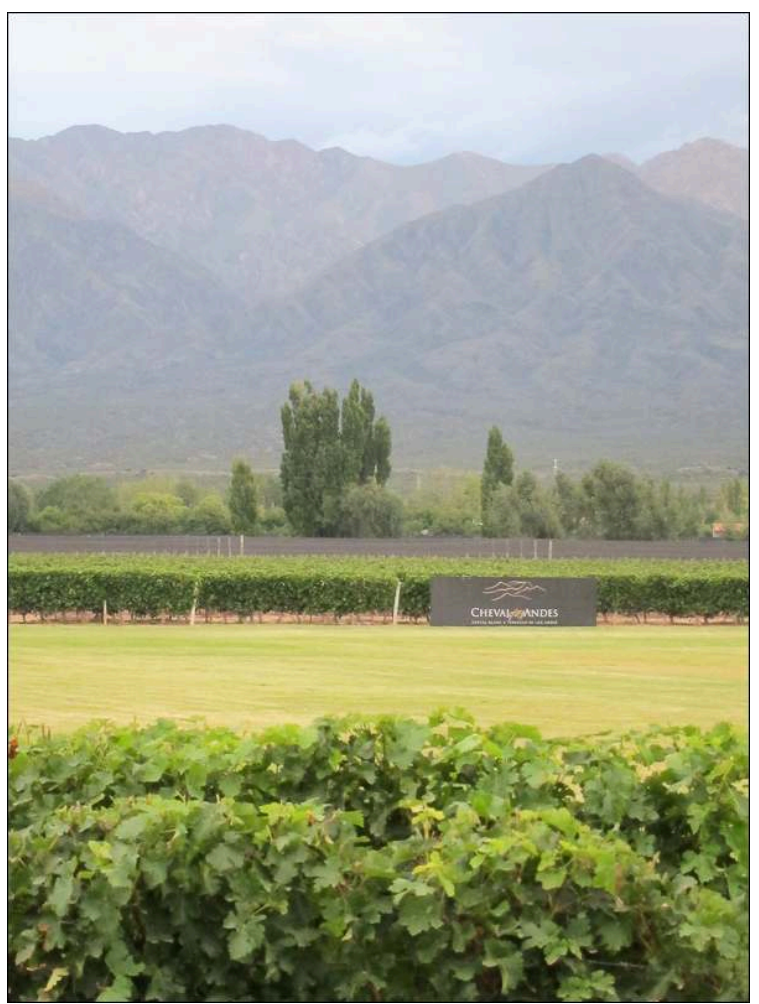

Auteur : J. Le Gall, 2011.

7 Le décor andin est utilisé par les bodegas et les acteurs touristiques pour mettre en avant la localisation originale de leur vignoble. En témoigne le nom des vins, telle la marque "Cheval de Los Andes », que présente ce cliché, version latino-américaine du Cheval Blanc bordelais créée par le groupe Moët et Chandon en association avec le domaine argentin «Terrazas de los Andes ». En témoignent également les étiquettes des bouteilles qui présentent un dessin, une photographie des Andes ou sont découpées suivant la forme d'une ligne de crête stylisée. En témoignent enfin les brochures touristiques qui utilisent systématiquement cette image pour présenter leurs circuits au cœur des vignes. Ces cartes postales sont un outil de mise en valeur de l'identité latine et andine des vins argentins et chiliens exportés à travers le monde. Mais elles servent surtout la publicité de grands groupes viticoles (Norton, Chandon) qui profitent de ces vins pour affirmer leur dimension internationale ou d'autres entreprises, qui utilisent ces endroits comme espaces de représentation. 


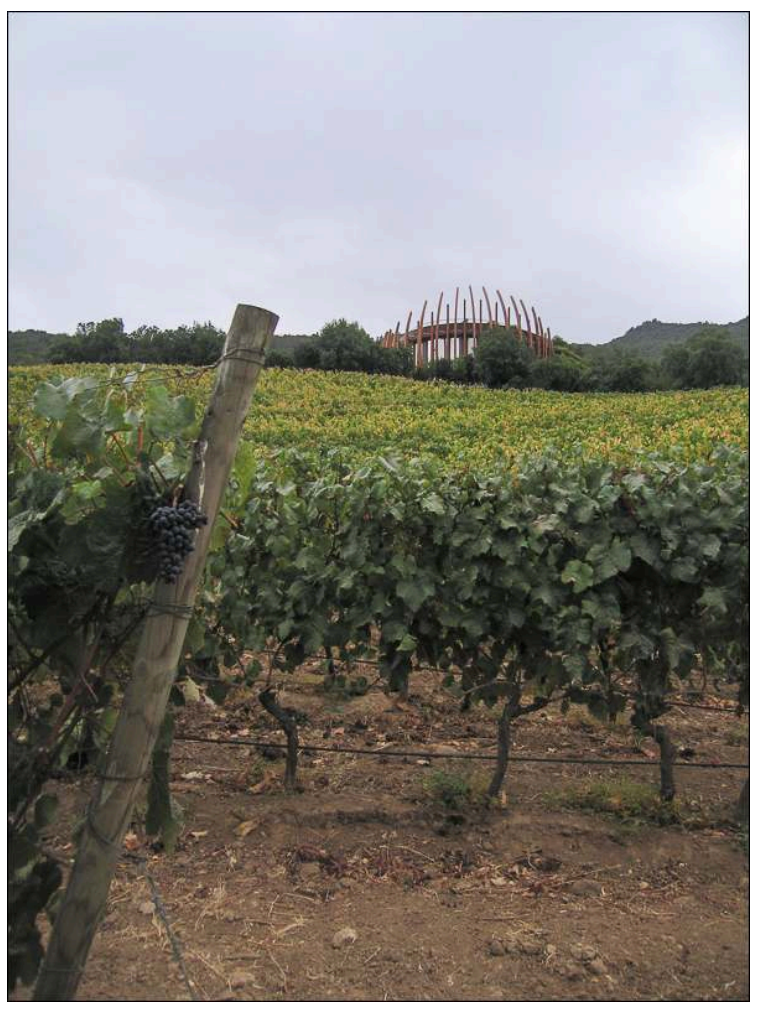

Auteur : C. Faliès, 13/04/12

8 Par-delà l'unité andine, les paysages que ces vignobles dessinent ont été profondément reconfigurés ces dernières années par l'importation de capitaux et acteurs extérieurs. Ainsi, en 1994, Alexandra Marnier Lapostolle, descendante de l'inventeur du Grand Marnier et par ailleurs présidente du groupe éponyme décide de constituer un vignoble dans la vallée de Colchagua à $200 \mathrm{~km}$ au sud de Santiago. Le clou de sa propriété et de sa production est le domaine Clos Apalta dont l'architecture a été confiée au célèbre architecte Roberto Benavente. Inaugurée en 2006, la bodega est constituée de quatre niveaux inspirés des tonneaux de collection acquis par la propriétaire et permet une vinification gravitationnelle : une terrasse ouverte panoramique permettant d'admirer les 350 hectares de vigne ; un niveau intermédiaire permettant de recevoir le raisin, de le trier et de le mettre dans des cuves de décantation situées derrière la baie vitrée visible sur la photo ; un rez-de-chaussée et une cave creusée dans la roche afin d'éviter la climatisation du site pour accueillir les fûts de chêne. Selon le principe des wineries californiennes cette architecture permet la vinification mais également l'accueil des touristes et l'organisation de dégustations très importantes pour l'image de marque du domaine. Le bâtiment que l'on aperçoit à gauche de la cave est l'un des quatre lodges créés par la propriétaire pour accueillir un tourisme de luxe : chacun porte un nom de cépage et ils figurent désormais dans le fameux guide des Relais et Châteaux. 
Illustration 4 - Bodega Viu Manent dans la vallée de Colchagua, Chili

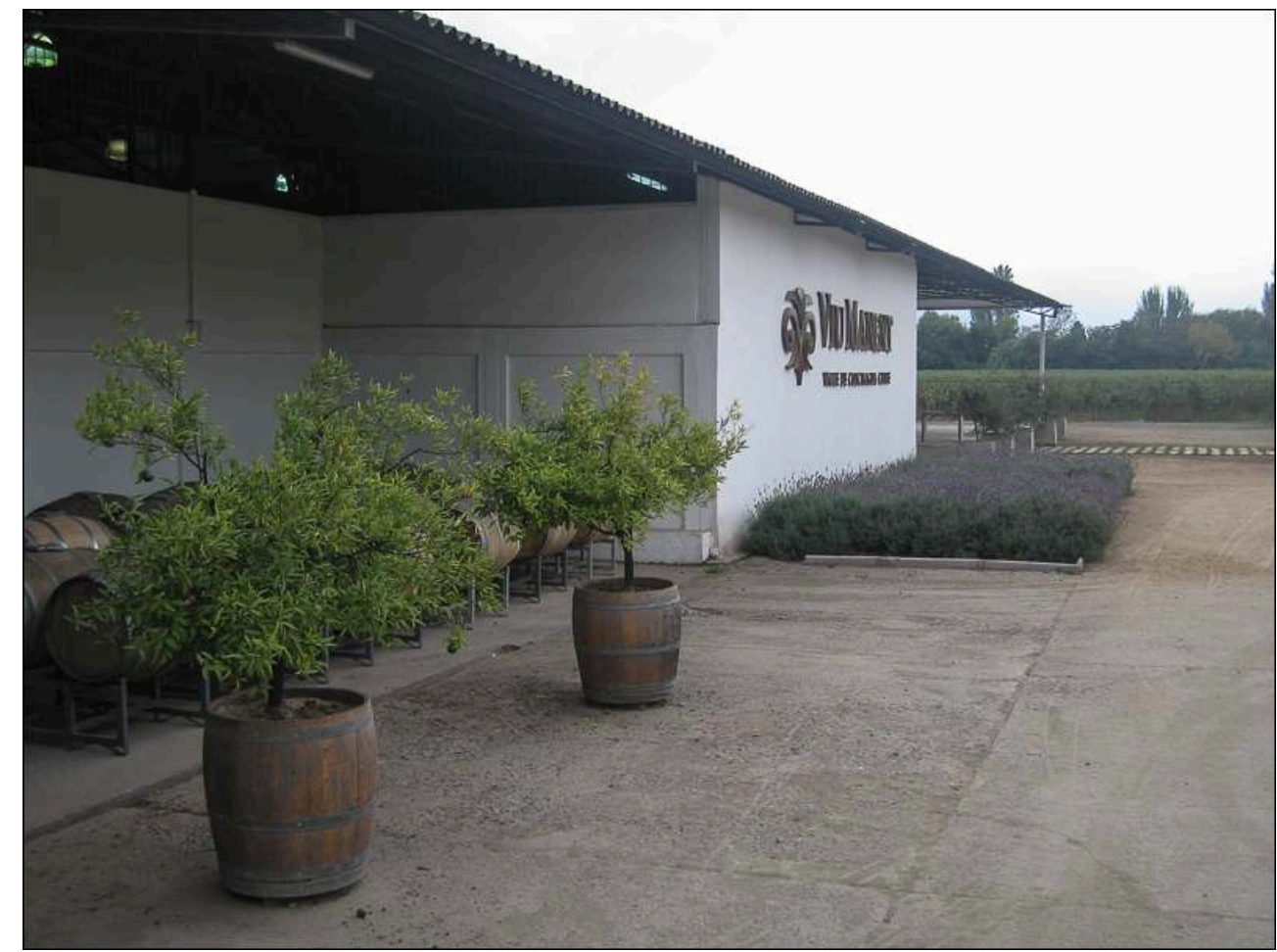

Auteur : C. Faliès, 13/04/12.

Illustration 5 - Salle de réception de la Bodega Cheval de los Andes, Mendoza

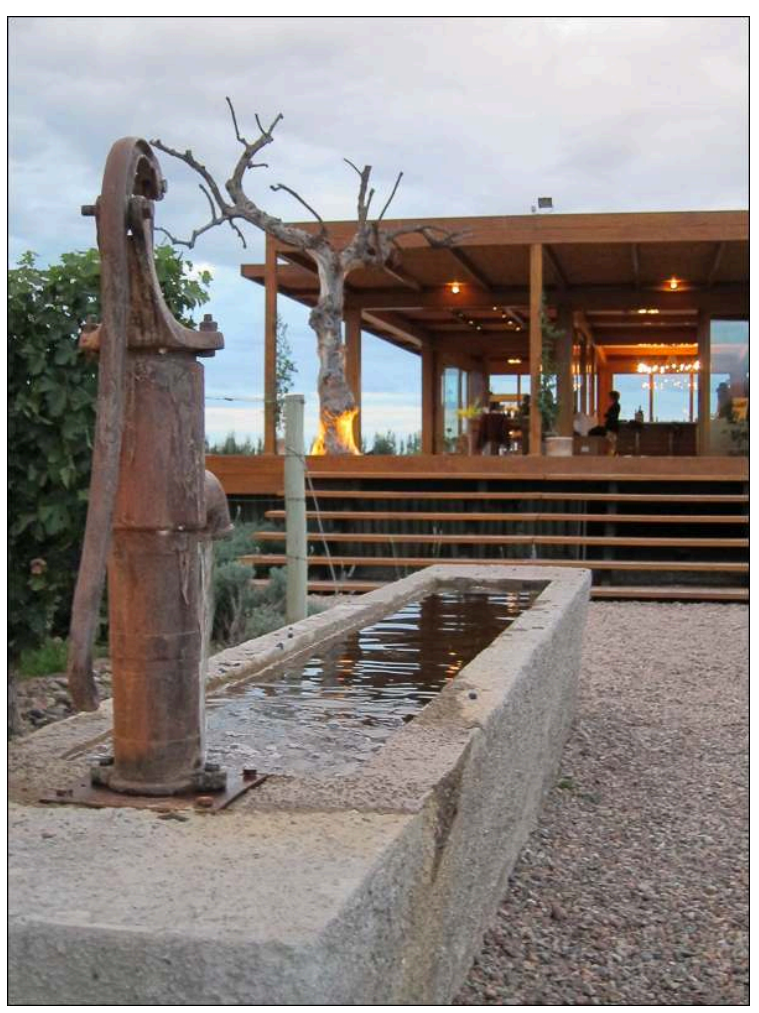

Auteur: J. Le Gall, 2011. 
L'aménagement des lieux et des bâtiments est parfois aussi important que l'attention portée aux pieds de vigne. Le caractère ancien ou au contraire moderniste et épuré de l'architecture répond à plusieurs objectifs. Cunaco datant du XIX siècle rachetée en 1966 par la famille Viu Manent d'origine
catalane. Ce bâtiment a été construit pour accueillir le laboratoire de vinification et stocker la production, bien que le maître de chai affirme que toute la production est achetée avant même la récolte et donc livrée dès le vin prêt. Il protège la production de l'aléa sismique plus que dans des caves anciennes qui menacent parfois de s'écrouler (cas de celle du Clos Ouvert lors du tremblement de terre de 2010). De même que le décor entourant le pavillon de bois qui trône au milieu des vignes de cheval de los Andes, le bâtiment de la bodega Viu Manent sert aussi à recréer une appartenance méditerranéenne: vieille fontaine, parterre de lavande, oliviers, tonneaux... En Argentine et au Chili, exhiber ses tonneaux français (Tonnellerie d'Aquitaine, Vicard, Sylvabois) est devenu très courant depuis quelques années. En effet, un usage excessif des copeaux de bois dans des cuves en acier pour feindre un élevage en fûts de chêne et pour coller au style Parker qui a longtemps promu des vins boisés et tanniques est régulièrement dénoncé. Ces tonneaux français rattachent donc les bodegas les plus prestigieuses à une certaine idée de la tradition française comme la cave clos Apalta qui expose lors de sa visite plusieurs tonneaux réalisés par le tonnelier Sylvabois à partir du chêne pluriséculaire de Bangor coupé en 2007 en forêt de Compiègne. C'est dans ce paysage pastiche du berceau de la vitiviniculture qu'ont enfin lieu les dégustations, prétextes également à d'autres manifestations: signatures de contrat, séminaires d'entreprise, expositions d'art,... Le pavillon de la Bodega Cheval de los Andes surplombe ainsi un terrain de polo créé à la demande de l'œnologue. Des matches animent les réceptions sur fond de musique française et visent à transmettre aux clients l'esprit de la maison. Dans cet univers, la personne la plus importante n'est pas tant le récoltant, mais plutôt l'œnologue à la tête du laboratoire et plus encore peut-être le consommateur comme R. Dion l'a démontré (Dion R., 1959). Le vin, le produit final, semble plus mis en valeur que la terre qui l'a façonné. Les fincas et les bodegas constituent donc non seulement des lieux de production mais surtout des vitrines.

Illustration 6 - Vieilles vignes franches de pied (non greffées) de cépage pré-phylloxériques dans la vallée du Maule

EchoGéo, 23 | 2013 


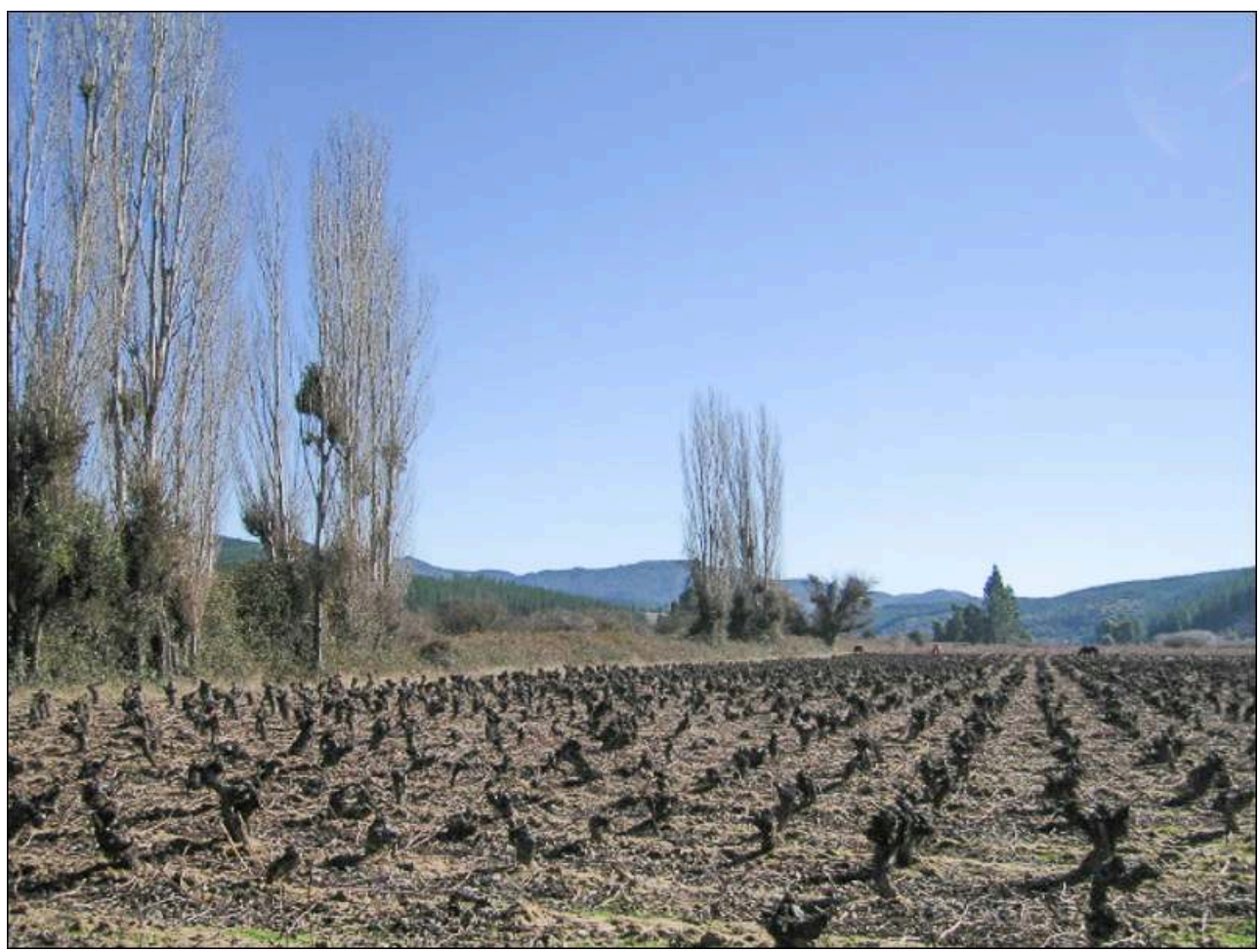

Auteur : C. Faliès, 27/07/2007.

11 Néanmoins, cette image unifiée et policée cache d'autres réalités. Des vignobles familiaux subsistent ou ont été mis en valeur parallèlement à l'arrivée des autres acteurs. Pour trouver leur place au sein d'un marché de plus en plus concentré et uniformisé, les exploitants font le choix de la diversification des productions ou de l'innovation des modes de production.

Louis-Antoine Luyt, un Français, a racheté ces vignes dans le but de produire un vin naturel à partir de ces "cépages pays ", comme on les appelle au Chili, dont la taille courte ainsi que l'absence d'irrigation nous indiquent bien qu'on ne se situe plus en contexte méditerranéen. Les vins du Clos Ouvert sont dits naturels car la culture des vignes se fait sans engrais chimiques ni désherbants et que la vinification ne fait appel ni au soufre ni aux levures. Jusqu'ici ces cépages servaient au mieux à produire de la "chicha", un jus de raisin légèrement fermenté et mousseux quand leurs vignes n'étaient pas complètement abandonnées.

Bien que minoritaires, ces dynamiques véhiculent une autre approche de la culture de la vigne et de la production de vin, qui entraîne une autre production de paysages.

Les grands groupes et investisseurs étrangers ont donc joué un grand rôle dans la configuration actuelle des paysages viticoles du Cône Sud. Depuis les années 1980-1990, ils ont participé à l'amélioration de la qualité des vins en travaillant sur les choix de cépage, la forme des vignes, la technification des systèmes d'irrigation et de la vinification, la modernisation des caves. Ces processus ont contribué à produire des paysages viticoles mondialisés faussement méditerranéens qui se ressemblent les uns les autres. Cependant, on ne peut pas parler uniquement de paysages importés. Le poids des exportations et le point de vue européen d'où l'on se place tendent à réduire notre perspective à l'image que les investisseurs les plus actifs et les acteurs touristiques ont utilisée pour se faire (re)connaître. D'autres dynamiques se font jour, 
chez les exploitants familiaux comme chez les grands groupes, afin de se distinguer et de conquérir de nouveaux marchés nationaux ou internationaux (marchés de niche). Elles impliquent parfois de repousser les limites de la vigne au-delà de ce qui s'est fait jusqu'à présent en installant de nouveaux réseaux d'irrigation ou montant davantage en altitude.

En conclusion, les vignobles chiliens et argentins sont donc bien plus divers que le film Mondovino n'a pu le laisser paraître. Loin de n'être que des héritages ou des plagiats de vignobles européens, ils connaissent de surcroît une diversification paysagère actuelle du fait d'un accroissement de la demande. Les limites de la vigne sont repoussées de plus en plus loin en altitude et en latitude relativisant définitivement les notions de « terroir » ou de « climats ».

16 Néanmoins, cette diversification est plus accentuée au Chili qu'en Argentine du fait de la production de raisins Moscatel à destination du pisco comme le montrent les illustrations 7 et 8 . Ces paysages réputés parmi les plus beaux du Chili pour l'encaissement des vallées, l'altitude et la présence d'une irrigation ancienne se transforment depuis les années 1980 et la conquête des versants et des fonds de vallée par la culture de la vigne non pour la production vinicole mais pour la distillation d'eau-de-vie.

17 D'une certaine manière ici, le paysage est préexistant à la vigne et se rattache davantage au contexte régional aride notamment en se comparant au voisin péruvien (palmiers de la finca, constructions basses, choix du toponyme Pisco) plutôt qu'en revendiquant un héritage méditerranéen. L'organisation ne se fait désormais plus autour de la bodega mais de la distillerie dans la vallée de l'Elqui. Les artefacts comme ces atrapanieblas visibles sur l'illustration 7 et qui permettent de recueillir les gouttes de brume condensées pour compenser une hydrométrie très faible sous ce climat aride ainsi que la finca ne constituent donc que des attraits paysagers supplémentaires au spectacle qu'offre la vallée. 
Illustration 7 - Vignoble de Pisco, Vallée de Colchiguaz, province de l'Elqui, 31 mai 2010

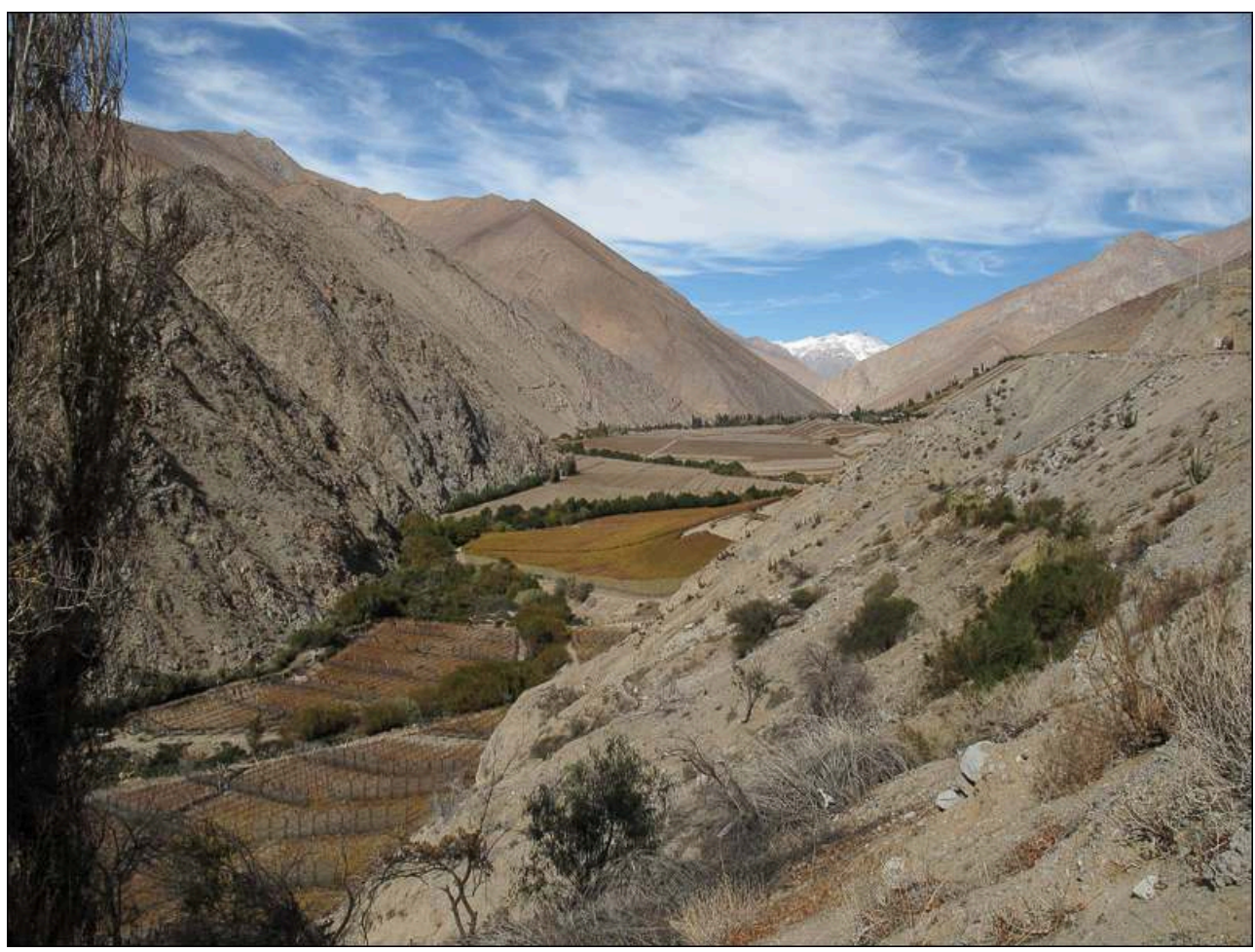

Auteur : C. Faliès, 31/05/2010.

Illustration 8 - Vignoble de Pisco, Vallée de Colchiguaz, province de l'Elqui, 31 mai 2010

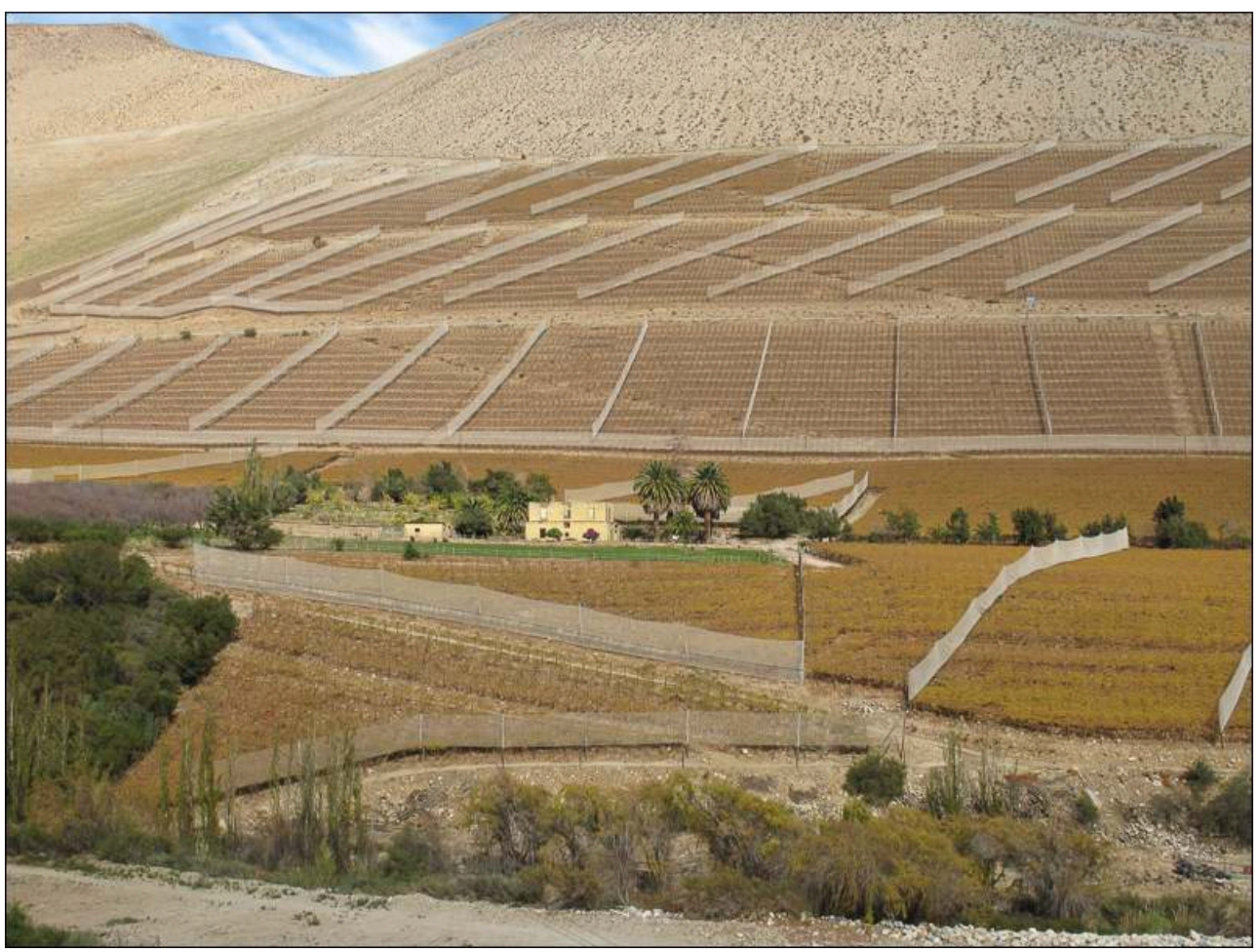

Auteur : C. Faliès, 31/05/2010. 


\section{BIBLIOGRAPHIE}

Crenn C., Techoueyres I., 2004. Vin et mondialisation », le point de vue d'un géographe [P. Roudié]. Anthropology of food, $n^{\circ} 3,12$ p.

Dion R., 1959. Histoire de la vigne et du vin en France, réédition CNRS, 2010, 772 p.

Schirmer R., 2007. Les vins du Nouveau Monde sont-ils a-géographiques. Bulletin de l'Association des Géographes Français, $\mathrm{n}^{\circ}$ 1, p. 65-80.

\section{AUTEURS}

\section{CÉCILE FALIÈS}

Cécile Faliès, cecilefalies@yahoo.fr, est Doctorante en géographie et membre de l'UMR 8685 Prodig, Université Paris 1-Panthéon Sorbonne.

\section{JULIE LE GALL}

Julie Le Gall, julie.legall@ens-lyon.fr, est Maître de conférences en géographie et membre de l'UMR 5600 EVS - équipe biogéophile, ENS de Lyon. 